\title{
Radio-activation of hTERT promoter in larynx squamous carcinoma cells: An 'indirected-activator' strategy in radio-gene-therapy
}

\author{
ZHENG-KAI LIAO, FU-XIANG ZHOU, ZHI-GUO LUO, WEN-JIE ZHANG, JIE XIONG, JIE BAO, \\ GUANG HAN, MING-SHENG ZHANG, CONG-HUA XIE and YUN-FENG ZHOU \\ Department of Cancer Radiochemotherapy, Wuhan University Zhongnan Hospital \\ and Cancer Research Center, Wuhan, Hubei 430071, P.R. China
}

Received July 30, 2007; Accepted September 21, 2007

\begin{abstract}
The usefulness of human telomerase reverse transcriptase (hTERT) gene promoter has been proposed in cancer-targeted gene therapy. However, this promoter may not be strong enough to achieve therapeutic levels of transgene expression. In this study, we tested an 'indirected-activator' strategy that utilizes radiation to increase the activity of the hTERT gene promoter. We demonstrated that hTERT may participate in the process of DNA repair induced by irradiation. We found that Zidovudine (AZT, an hTERT inhibitor) can decrease the telomerase activity in human HEp-2 larynx squamous carcinoma cells and lower the survival fraction of HEp-2 cells exposed to radiation. In HEp-2 cells exposed to 6 Gy-radiation, the hTERT promoter showed 2.9-fold higher activity compared with unirradiated cells. Importantly, an increased expression of enzyme horseradish peroxidase (HRP) controlled by the hTERT promoter was found in the transfected cells after irradiation, which coincided with a higher killing rate for HEp-2 cells after prodrug indole3 -acetic acid (IAA; converted by HRP into a cytotoxin) incubation combined with irradiation or not. Our observations suggest that hTERT promoter-mediated gene therapy could be improved in combination with radiotherapy, which may be due to cellular DNA damage responses.
\end{abstract}

Correspondence to: Dr Yun-Feng Zhou, Department of Radiochemotherapy, Zhongnan Hospital, Wuhan University, 169 Dong Hu Road, Wuhan, Hubei 430071, P.R. China

E-mail: yfzhouwhu@gmail.com

Abbreviations: AZT, Zidovudine; 3'-azido-3'-deoxythymidine; GDEPT, gene directed enzyme prodrug therapy; hTERT, human telomerase reverse transcriptase; HRP, horseradish peroxidase; IAA, indole-3-acetic acid; SER, sensitization enhancement ratio

Key words: gene therapy, radiotherapy, human telomerase reverse transcriptase promoter, horseradish peroxidase/indole-3-acetic acid, laryngeal squamous cell carcinoma

\section{Introduction}

Telomeres play an important role in maintaining chromosomal integrity and function. Telomerase, responsible for maintaining telomere function through the addition of telomere repeats to chromosome ends, is a ribonucleoprotein complex and is activated in most cancer cells, but not in most normal cells (1). Human telomerase reverse transcriptase (hTERT) is the catalytic subunit of telomerase that determines the telomerase activity (2). Thus, the hTERT gene promoter has been widely used in gene therapy for targeted cancer cells (3). However, the promoter activity may not be strong enough to induce the expression of a significant amount of a therapeutic gene product required to kill cancer cells $(3,4)$.

Radiotherapy is one of the major clinical options for malignant tumors, while cellular DNA damage responses are triggered when cells are exposed to irradiation. Previous studies have reported an initial increase of telomerase activity after irradiation in human colon carcinoma, lymphoma, myeloma cells and Ewing xenograft tumors (5-8). We previously observed the up-regulation of telomerase activity in human U251 malignant glioma cells after 2 Gy-radiation exposure, which may be a reaction to DNA damage induced by irradiation. Our data suggested a role of telomerase in the healing of chromosome breaks by radiation-induced DNA damage (9). The above suggests that precise irradiation may benefit targeted enhancement of hTERT promoter activity, which is promising in targeted cancer gene therapy.

In this study, we examined the usefulness of a strategy, referred to as 'indirected-activator' strategy, in potential radio-gene-therapy. The strategy uses radiation as a first activator to induce DNA damage and in turn, cellular DNA damage responses serve as a second activator to enable an enhancement of hTERT promoter activity. We treated HEp-2 cells with an hTERT inhibitor, Zidovudine (AZT), and then telomerase activity and clonogenic survival fraction were measured after irradiation to understand the mechanism (s) of the increased activity. In addition, we evaluated the radiationinduced activities of hTERT promoter and telomerase. Furthermore, horseradish peroxidase (HRP)/indole-3-acetic acid (IAA) gene directed enzyme prodrug therapy (GDEPT) system was used to determine whether the combination of 
irradiation and the hTERT promoter-controlled gene therapy was applicable to cancer gene therapy.

\section{Materials and methods}

Cell culture. The human HEp-2 larynx squamous carcinoma cell line (ATCC CCL-23) was obtained from the China Center for Type Culture Collection. Cells were cultured in RPMI-1640 medium supplemented with $10 \%$ calf serum (Gibco) and maintained at $37^{\circ} \mathrm{C}$ with $5 \% \mathrm{CO}_{2}$ in a humidified incubator.

Plasmid DNA and cell transfection. The core hTERT promoter $(-385 /+40$ bp) was kindly provided by Dr I. Horikawa (NIH/NCI) (10). For phTERTp-Luc, the promoter was placed upstream of the luciferase gene in the pGL3-Basic vector (Promega). For phTERTp-MCS, which was used as a negative control since there was no gene expression controlled by the hTERT promoter, the promoter from pcDNA3.1(-) (Invitrogen) was replaced with the hTERT promoter. The plasmid pRK5HRP was a kind gift from Dr D.F. Cutler (UCL) (11). The HRP cDNA was excised from pRK5-HRP by BamHI digestion (enzymes from Takara) and the resulting fragment was inserted into the multiple cloning site (MCS) directly downstream of the hTERT promoter in the plasmid phTERTp-MCS. Transient cell transfectants for all plasmid constructs were produced by using Metafectene ${ }^{\mathrm{TM}}$ (Biontex) according to the manufacturer's guidebook.

Reporter gene assay. Plates (6-well) were seeded with $4 \times 10^{5}$ cells/well. At an optical confluency of $90-100 \%$, cells were cotransfected with $0.5 \mu \mathrm{g}$ of plasmid phTERTp-Luc or pGL3-Basic (negative control plasmid, Promega) and $0.5 \mu \mathrm{g}$ of pRL-TK (cotransfected standard plasmid, Promega). Cells were irradiated after transfection for $24 \mathrm{~h}$ and lysed for luciferase activity analyses at different time points postradiation, using the Dual-luciferase reporter assay system (Promega) as indicated by the manufacturer. Internal normalization of the transfection efficacy was performed using the value of Renilla luciferase (RL). The value of luciferase activity in negative control cells (cotransfected pGL3-Basic and pRL-TK) reflected the background, which were deducted.

Analysis of telomerase activity. Telomerase activity was determined using the TRAP PCR ELISA kit (Roche) as recommended by the manufacturer. Briefly, cell extracts were obtained from $2 \times 10^{5}$ cells lysed with $200 \mu 1$ lysis buffer, incubated on ice for $30 \mathrm{~min}$, and centrifuged at $16,000 \mathrm{~g}$ at $4^{\circ} \mathrm{C}$ for $20 \mathrm{~min}$. Protein concentration was determined by the commercial BCA protein assay kit (Beyotime). Each Trap reaction contained $50 \mu \mathrm{g}$ total protein. The primer was elongated at $25^{\circ} \mathrm{C}$ for $30 \mathrm{~min}$, and then incubated at $94^{\circ} \mathrm{C}$ for 5 min to induce the telomerase inactivation. The reaction mixture was then amplified by 30 cycles of polymerase chain reactions (PCR) at $94^{\circ} \mathrm{C}$ for $30 \mathrm{sec}, 50^{\circ} \mathrm{C}$ for $30 \mathrm{sec}, 72^{\circ} \mathrm{C}$ for $90 \mathrm{sec}$, and $72^{\circ} \mathrm{C}$ for $10 \mathrm{~min}$ for the final step. The amplification product $(5 \mu \mathrm{l})$ was transferred for hybridization and ELISA procedure. Sample absorbance was measured with Model 550 microplate reader (Bio-Rad, USA) at the wavelength of $450 / 690 \mathrm{~nm}$ within $30 \mathrm{~min}$ after addition of the stop reagent. Each assay contained a negative and positive control included in the kit. All experiments were performed twice (triplicate samples) to ensure the reliability of the test.

Reverse transcriptase-PCR analysis. Total RNA was isolated from cells using TRI Reagent (MRC). First-strand cDNA was synthesized from $2 \mu \mathrm{g}$ of RNA in $20 \mu \mathrm{l}$ of reaction solution using the Revert aid first-strand cDNA synthesis kit (Fermentas). The hTERT cDNA, was amplified in a PCR reaction using the primers 5'-CTGCCGTCTTCACTTCCC CAC-3' (forward) and 5'-TTACTCCCACAGCACCTCCCC-3' (reverse) designed accordly to complete hTERT mRNA (NM_198253). The HRP cDNA (J05552) was amplified using the primers 5'-CCTTGTGGCTCTGTCCGGAGGA-3' (forward) and 5'-AGTGGGATGGTGTCAGTGGCGT-3' (reverse). As a control, we amplified the GAPDH cDNA (NM_002046) with the following primers: 5'-ACCACAGTC CATGCCATCAC-3' (forward) and 5'-TCCACCACCCTGT TGCTGTA-3' (reverse). The thermal cycling condition for hTERT PCR was: $94^{\circ} \mathrm{C}$ for $5 \mathrm{~min}$, followed by 35 cycles of $94^{\circ} \mathrm{C}$ for $30 \mathrm{sec}, 64^{\circ} \mathrm{C}$ for $40 \mathrm{sec}$, and $72^{\circ} \mathrm{C}$ for $1 \mathrm{~min}$. For HRP PCR: $95^{\circ} \mathrm{C}$ for $3 \mathrm{~min}$, followed by 30 cycles of $94^{\circ} \mathrm{C}$ for $1 \mathrm{~min}, 65^{\circ} \mathrm{C}$ for $1 \mathrm{~min}$, and $72^{\circ} \mathrm{C}$ for $1 \mathrm{~min}$. The PCR products were resolved on a $2.5 \%$ agarose gel and stained with ethidium bromide (Sigma). The average band densities of the PCR products were measured using Genetools software (Syngene). All experiments were repeated three times.

Western blotting. Western blot analysis for HRP expression was performed as described previously (16). Briefly, HEp-2 cells that had been transfected with plasmid phTERTp-HRP or phTERTp-MCS (negative control) were washed with PBS and lysed in a protein extraction buffer according to the manufacturer's protocols (Beyotime). Cell lysates were collected, separated by $12 \%$ SDS-PAGE gels and transferred to nitrocellulose membranes (GE). The blocked membranes were then incubated with a primary antibody against HRP (Bioss) and then with the corresponding peroxidase were labeled as a secondary antibody (Pierce). Immunoreactive proteins were visualized using the DAB staining kit (Zsbio) according to the instructions provided. Glyceraldehyde 3phosphate dehydrogenase (GAPDH) was used as a protein loading control.

Clonogenic assays. The effect of AZT on radiosensitivity of HEp-2 cells and the cytotoxicity of phTERTp-HRP/IAA GDEPT after activation by a radiation trigger were examined by clonogenic survival assays. After different treatments, indicated in the figure legends, the cells were cultured for 14 days. After fixation and staining with $1 \%$ crystal violet (Sigma) w/v in dehydrated alcohol, colonies of $>50$ cells were scored. The plating efficiency and survival fraction were calculated. The survival curves were obtained and analyzed with SPSS 11.0 statistical software to get the surviving fractions at 2 Gy-radiation $\left(\mathrm{SF}_{2}\right)$ of each group. Sensitizer enhancement ratio (SER) was defined as the ratio of the radiation dose required to obtain a specific surviving fraction $(\mathrm{SF})$.

Statistical analyses. The data were expressed as mean \pm standard deviation. Significance tests were carried out on the 

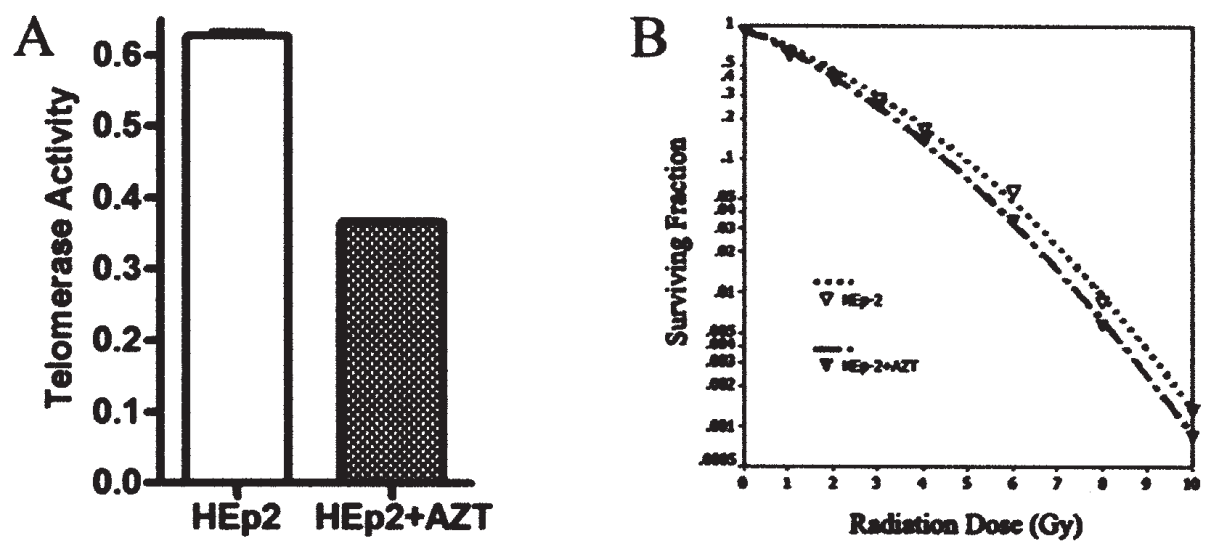

Figure 1. Effect of AZT on telomerase activity and radiosensitivity of HEp-2 cells. Exponentially growing cells plated in $25 \mathrm{~cm}^{2}$ culture flasks were divided into 2 groups: i) HEp-2+AZT: with incubation in $0.8 \mathrm{mM}$ AZT for $24 \mathrm{~h}$; ii) HEp-2: with mock-incubation for $24 \mathrm{~h}$. Then, the cells were collected to measure telomerase activities. (A) Telomerase activity in HEp-2 cells with or without AZT treatment. (B) Radio-sensitivity of HEp-2 cells with or without AZT treatment. After incubation, cells were treated with various doses $(0,1,2,3,4,6,8,10 \mathrm{~Gy})$ of ${ }^{60} \mathrm{Co} \gamma$-rays $\left({ }^{60} \mathrm{Co}\right.$ therapeutic machine, GWXJ80 type, Chengdu, China. Dose rate $=61.22 \mathrm{cGy} / \mathrm{min}$, Source to surface distance $80 \mathrm{~cm}$ ). Cell survival fractions were normalized for the plating efficiency of non-irradiated cells exposed to AZT or not, to account for its cytotoxicity.
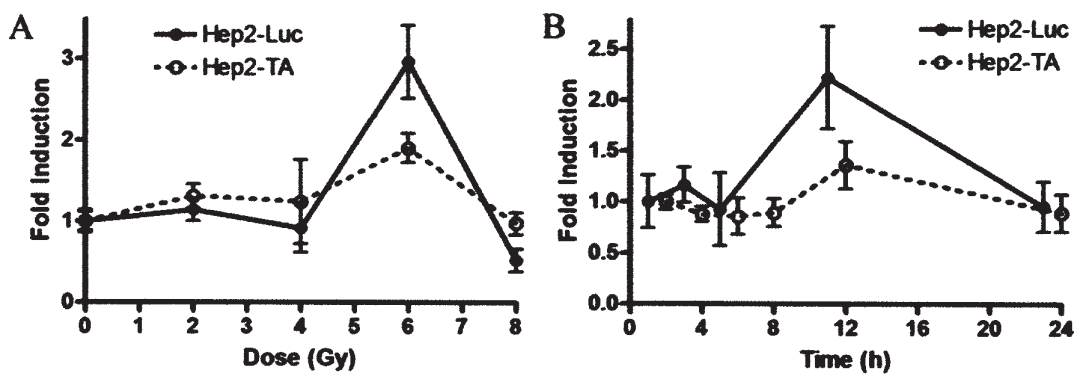

Figure 2. Exogenous hTERT promoter activity and endogenous telomerase activity after radiation in HEp-2 cells. Exogenous hTERT promoter activity was evaluated by luciferase activity assay. Plasmids phTERTp-Luc or pGL3-Basic (negative control plasmid) were transiently cotransfected with pRL-TK (cotransfected standard plasmid) into HEp-2 cells. Telomerase activity was measured using the TRAP PCR ELISA kit. (A) The cells were irradiated with different doses $(0,2,4,6,8 \mathrm{~Gy})$ of radiation and collected at 24-h post-exposure time. (B) The cells were irradiated with 2 Gy $\gamma$-radiation and collected at different time points postradiation. The exogenous hTERT promoter activity and endogenous telomerase activity were expressed as fold induction relative to those of 0 Gy treatment (A) or $0 \mathrm{~h}$ post-radiation (B). The means of at least two experiments (triplicate samples) \pm SD are shown.

date groups by independent-samples t-test. Differences among the treatment groups were analyzed by the one-way ANOVA test. Differences between the survival fractions of the treatment groups were analyzed by the Chi-squared test. Correlations were assessed by bivariate correlation analysis. The data were analyzed using SPSS11.0 software (SPSS Inc., USA). $\mathrm{P}<0.05$ was considered statistically significant.

\section{Results}

Decrease of telomerase activity and increase of radiosensitivity by AZT. We favor the hypothesis that telomerase may participate in the process of DNA repair and the up-regulation of telomerase may be a reaction to DNA damage induced by irradiation. In previous work, we observed that AZT (an hTERT inhibitor) could diminish the repair of the DNA strand breaks after irradiation and radiosensitize U251 cells (9). Here, we found that AZT decreased the telomerase activity in HEp-2 cells and lowered the survival fraction of HEp-2 cells exposed to radiation (Fig. 1). After $0.8 \mathrm{mM}$ AZT incubation for $24 \mathrm{~h}$, a significant down-regulation in telomerase activity was observed in HEp- 2 cells $(0.627 \pm 0.015$ vs. $0.368 \pm 0.003$,
$\mathrm{P}<0.01$, Fig. 1A), which indicates that AZT suppressed the telomerase activity of the HEp-2 cells. Data showed that radiation alone caused a dose-dependent reduction and that HEp-2 cells were more radiosensitive after AZT treatment in cell survival (Fig. 1B). SF 2 of HEp-2 cells dropped from 0.4147 (HEp-2 group) to 0.3843 (HEp-2+AZT group), $\operatorname{SER}_{2 \mathrm{~Gy}}=1.079\left(\chi^{2}=63.957, \mathrm{P}<0.01\right)$.

Regulation of exogenous hTERT promoter activity and endogenous telomerase activity by irradiation. In an effort to understand whether the exogenous hTERT promoter activity would be modulated by radiation and potential usage in controlling exogenous gene, the firefly luciferase was used as the reporter gene to determine the instantaneous activation of hTERT promoter after $\gamma$-ray exposure. Renilla luciferase (RL) was used for internal normalization and pGL3-Basic was used as a negative control and background value. The effects of single-dose radiation on endogenous telomerase activity were also studied to determine whether the level of telomerase activity would be modulated by radiation. hTERT promoter activity and telomerase activity varied at different dose levels and different time points post-irradiation in the HEp- 2 cells 

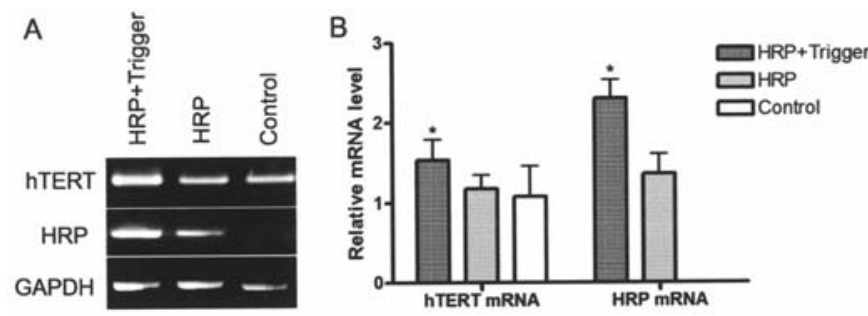

Figure 3. Effect of 6 Gy-radiation (Trigger) on expression of endogenous hTERT mRNA and exogenous HRP mRNA. HEp-2 cells were divided into three groups: i) HRP+Trigger: Cells transfected with plasmid phTERTp-HRP. Twenty-four hours after transfection, cells were exposed to 6 Gy-radiation (Trigger) and harvested $24 \mathrm{~h}$ later; ii) HRP: Cells transfected with plasmid phTERTp-HRP and treated with mock-irradiation; iii) control: Cells transfected with negative control plasmid phTERTp-MCS and treated with mock-irradiation. (A) Representative endogenous hTERT mRNA and exogenous HRP mRNA was analyzed by RT-PCR. GAPDH was used as an internal control. (B) Graphical representation of data shown in A. The densities of hTERT and HRP bands were compared with those of GAPDH controls and the results were expressed as density ratios. The means of three experiments $\pm \mathrm{SD}$ are shown. The relative mRNA levels were compared to the HRP+Trigger group. Statistically significant differences $\left({ }^{*} \mathrm{P}<0.05\right)$.

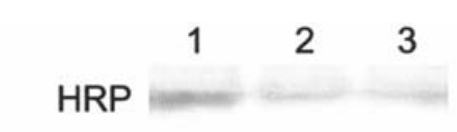

$\beta$-Actin

Figure 4. Representative HRP protein expression was detected in HEp-2 cells by Western blotting. $\beta$-actin was used as a control. Lane 1, HRP+Trigger group: Cells transfected with plasmid phTERTp-HRP. Twenty-four hours after transfection, cells were exposed to 6 Gy-radiation (Trigger) and harvested $24 \mathrm{~h}$ later; lane 2, HRP group: Cells transfected with plasmid phTERTp-HRP and treated with mock-irradiation; and lane 3 , control: transfected with negative control plasmid phTERTp-MCS and treated with mock-irradiation.

transfected with plasmid phTERTp-Luc (Fig. 2). A range of radiation doses, from 0 to $8 \mathrm{~Gy}$, was chosen according to the hTERT promoter activity and telomerase activity was measured $24 \mathrm{~h}$ after irradiation. As shown in Fig. 2A, there was an increased hTERT promoter activity after cells were treated with relatively low doses of radiation (2-6 Gy), which resulted in 1.31-fold to 1.89 -fold increased telomerase activity compared to the untreated controls ( 0 Gy group). The peak of the hTERT promoter activity $(2.96 \pm 0.45$-fold increase) was found in cells treated with 6 Gy-radiation, which resulted in telomerase activity of 1.89 -fold increase (Fig. 2A). In order to prove the induction of hTERT promoter by radiation, 6 Gy-radiation was adopted for the following experiments. The hTERT promoter activity started to increase at $3 \mathrm{~h}$ post-irradiation and reached a peak $(2.22 \pm 0.50$-fold increase) at $\sim 11 \mathrm{~h}$ after irradiation, which resulted in the maximal increasing telomerase activity (1.36 \pm 0.23 -fold increase) (Fig. 2B). Induced activity of hTERT promoter was shown in a dose- and time-dependent manner as that of telomerase activity $(\mathrm{r}=0.958, \mathrm{P}<0.05$, Fig. $2 \mathrm{~A} ; \mathrm{r}=0.950, \mathrm{P}<0.05$, Fig. $2 \mathrm{~B}$ ), which indicated that exogenous hTERT promoter activity reflected the difference in endogenous telomerase activity.

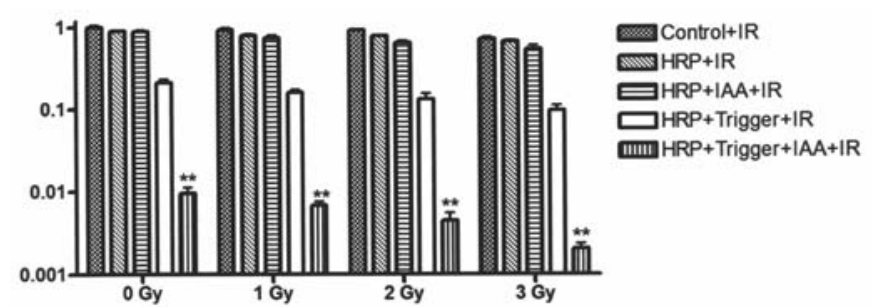

Figure 5. Combination of 6 Gy-radiation (Trigger) and phTERTp-HRP/IAA leads to the lowest surviving fraction. Exponentially growing cells plated in $25 \mathrm{~cm}^{2}$ culture flasks were divided into mock-transfected cells (control) and transfected cells. Cells transfected with plasmid phTERTp-HRP were divided into four groups: i) HRP+Trigger+IAA+IR: After transfected for $24 \mathrm{~h}$, cells were treated with 6 Gy-radiation (Trigger). Twenty-four hours later, cells were exposed to a culture medium containing $0.5 \mathrm{mM}$ IAA resolved in phenol red-free Hanks balanced salt solution (HBSS) for $24 \mathrm{~h}$. Then, cells were treated with a single-dose radiation $(0,1,2,3$ Gy); ii) HRP+Trigger+IR: cells were treated like group 1 except for a mock IAA incubation, in which cells were exposed to a culture medium supplemented with $10 \%$ foetal calf and HBSS without IAA; iii) HRP+IAA+IR: cells were treated like group 1 except for a mock-trigger; iv) HRP+IR: cells were exposed to mock-trigger and mock IAA incubation before the single-dose radiation. Surviving fraction was normalized for the plating efficiency of cells in the control group irradiated with 0 Gy-radiation. The means of two experiments (triplicate samples) $\pm \mathrm{SD}$ are shown. The surviving fractions were compared to the HRP+Trigger+AA+IR group exposed to the same single-dose radiation. Statistically significant differences $\left({ }^{* *} \mathrm{P}<0.01\right)$.

Irradiation enhances both the exogenous and endogenous hTERT promoter activity. In order to study the application of the hTERT promoter in gene therapy, the HRP/IAA system was adopted for use in the hTERT promoter-mediated gene therapy combined with radiation. Enhancement of hTERT mRNA expression, which was controlled by the endogenous hTERT promoter, was performed after 6 Gy-radiation treatment (Fig. 3). The effective induction of the HRP mRNA expression, which was controlled by the exogenous hTERT promoter, was compared by RT-PCR in HEp-2 cells with complete absence in cells of the control group transfected with phTERTp-MCS (Fig. 3). Syntheses of HRP protein were confirmed by Western blotting (Fig. 4). According to the levels of HRP mRNA, 6 Gy-radiation had parallel effects on HRP protein expression, which showed a 1.9-fold increase within $24 \mathrm{~h}$ of irradiation. Detectable proteins were investigated in the control group (lane 3, Fig. 4), which may be due to the mammalian homologues of HRP as the HRP mRNA were absent (control, Fig. 3). HRP, isolated from the roots of a horseradish plant, is a heme enzyme and its active site resembles that of hemoglobin and myoglobin (13). Based on published amino acid sequence, the construct encoding HRP isoenzyme $\mathrm{C}$ was artificially constructed and incorporated into the plasmid by Cutler et al (11). Thus, there is no endogenous HRP in HEp-2 cells.

Radiation increases the cytotoxicity of phTERTp-HRP/IAA $G D E P T$. At the doses of 0.25 to $2 \mathrm{mM}$, cytotoxic effects of IAA (Fluka) were previously measured. No toxicity was detected in mock-transfected HEp-2 cells exposed for $48 \mathrm{~h}$ to up to $0.5 \mathrm{mM}$ IAA (data not shown), therefore $0.5 \mathrm{mM}$ was chosen. Prodrug IAA incubation was performed after trigger treatment, and the efficacy of the hTERT promoter induction was compared with a single component control after the 
HEp-2 cells were exposed to a single-dose radiation $(0,1$, 2, 3 Gy) (Fig. 5). To evaluate the application of this strategy, plasmids phTERTp-HRP were transiently transfected into HEp-2 cells. In the 0 Gy group, as expected, when the hTERT promoter alone was used to control HRP expression, HEp-2 cells were not killed after 0.5 mM IAA incubation for $24 \mathrm{~h}$ ( $\mathrm{p}>0.05)$, probably due to the low activity of the hTERT promoter. In addition, $21.1 \%$ of the HEp-2 cells survived after 6 Gy-radiation exposure. Conversely, when HRP expression controlled by the hTERT promoter combined with trigger (6 Gy-radiation), only $0.95 \%$ of HEp-2 cells survived after IAA incubation, which accorded with the up-regulated expression of HRP (Figs. 3 and 4) and indicated that stimulation of irradiation dramatically induced the activity of hTERT promoter. We investigated whether conventional treatments augment the efficacy of the combination of trigger and phTERTp-HRP/IAA GDEPT. The cells were exposed to a low-dose radiation (1-3 Gy) after treatments. As shown in Fig. 5, combination strategy dramatically increased the cytotoxicity induced by HRP/IAA GDEPT in each group. On the other hand, higher radiation doses show more significant combination effects with phTERTp-HRP/IAA. These results suggest that the cytotoxicity of phTERTp-HRP/ IAA is preferably combined with irradiation. Nonspecific activation of IAA in normal tissues is unlikely to take place, since IAA is a poor substrate for mammalian peroxidases (14). Endogenous myeloperoxidase (MPO) was notably less efficient than HRP in converting IAA into a cytotoxin at therapeutically significant prodrug doses (15). Thus, cytotoxin must come from exogenous HRP/IAA.

\section{Discussion}

We found that the activities of the exogenous hTERT promoter and endogenous telomerase in HEp-2 cells can be induced by irradiation. The up-regulated activity of both exogenous and endogenous hTERT promoters may be a reaction to DNA damage, which is one of the mechanisms involved in increased telomerase activity after irradiation. These observations indicate that the 'indirected-activator' strategy may be able to amplify the targeted killing effect of hTERT promoter mediated gene therapy due to the DNA damage response after irradiation.

Gene therapy approaches based on the selective expression of therapeutic genes present a number of distinct advantages. The targetability of radiation therapy offers a unique opportunity to control therapeutic gene expression (16). Some researchers have used promoters of radiation-inducible genes to drive the transcription of transgenes in the response to radiation. For example, the early growth response-1 (Egr-1) gene promoter, the first radiation inducible promoter system, was inducible by exposure to ionizing radiation via radical oxygen intermediate (ROI) formation and that the CArG (CC [AtT-rich] 6GG) domain of the promoter are the sequences necessary (17). In this study, we proved there is an 'indirected-activator' strategy which can be used in targeted gene therapy. The strategy uses radiation-induced DNA damage as a therapeutic tool and as an activator to enhance the activity of the hTERT promoter. This hypothesis may explain the satisfactory results obtained in few studies, which had combined radiotherapy with gene therapy using the hTERT promoter $(18,19)$.

There is increasing evidence that proteins involved in DNA damage response, including $\mathrm{Ku}$, DNA-PKcs, RAD51D, PARP-2, WRN and RAD50/MRE11/NBS1 complex directly participate in telomere maintenance. A close association between telomeres and different DNA damage response factors is a logical result of chromosomal integrity maintenance activities (20). Although the exact mechanism of DNA repair is not clear, many scholars believe that the activation of telomerase observed post-radiation may be due to the involvement of telomerase in DNA repair and chromosome healing $(21,22)$. In previous work, we observed that a reverse transcriptase inhibitor (AZT) suppressed the induction of the telomerase activity by $\gamma$-irradiation, decelerated the restored rate of shortened telomere, decreased the repair rate of the DNA strand breaks, and increased the radiosensitivity in U251 cells (9). In this assay, we know that telomerase also plays an integral role in DNA repair, since after AZT treatment, HEp-2 cells displayed an obvious decrease in telomerase activity and in DNA repair capacity shown as an increase of radiosensitivity. In addition, hTERT promoter activity, hTERT mRNA expression, and telomerase activity were up-regulated by irradiation, which suggests DNA damage response factors may increase telomerase activity by increasing hTERT expression at the promoter level and may be one of the mechanisms involved in radiosensitivity in tumor cell lines.

Although induced activity of the hTERT promoter was shown in both a time- and dose-dependent manner as that of telomerase activity, the increasing levels were not consistent in our study. The regulation mechanism of hTERT activation has not been confirmed yet. Few previous works have shown that hTERT activation subjects to transcriptional or posttranslational regulation, and that the telomerase activities and the hTERT mRNA levels in cancer cells were not always in parallel $(23,24)$. However, in this study, the radiation-induced response has been demonstrated at the expression level for exogenous hTERT promoter activity, endogenous telomerase activity, and hTERT mRNA controlled by endogenous hTERT promoter. We hypothesize that transcriptional regulation is essential to telomerase activity in cells with DNA damage after irradiation. Few cell lines have exhibited different regularities according to the time and doses used. For example, HeLa cells and ataxia-telangiectasia cells show a dose-dependent ( 0 to $40 \mathrm{~Gy}$ ) decrease in telomerase activity after ionizing radiation (25). The activity of the hTERT promoter and expression of hTERT mRNA may be more essential than telomerase activity in reflecting radiosensitivity. Since there may be some post-translational regulations that have negative effects on telomerase activity, additional studies in activity regulation of telemerase due to radiation are certainly required.

The usefulness of the hTERT promoter has been proven in targeted gene therapy, but the weak activity hinders its therapeutic applications. Radiotherapy is one of the main methods in cancer therapy. In the 'indirected-activator' strategy, precise radiotherapy was utilized to target enhanced therapeutic gene expression controlled by the hTERT promoter, which may achieve both high-selectivity and high- 
efficiency in cancer-targeted gene therapy. With a view to combining targeted gene therapy with conventional radiotherapy, an increase in therapeutic gain could be achieved both at the level of the promoter and the therapeutic gene (26). For example, the HRP/IAA system, a novel GDEPT system, exhibited a rapid efficiency in killing tumor cells with a strong bystander effect both in anoxic and oxic conditions and showed a significant synergistic effect with radiation $(12,27)$. Thus, this system is promising if it is to be used in combination with radiotherapy and gene therapy.

To determine whether the 'indirected-activator' strategy was applicable to cancer gene therapy, plasmid vectors were tested, in which the tumor-targeted and radiation-inducible hTERT promoter controlled the production of the enzyme HRP. HEp-2 cells were transiently transfected with these constructs and their sensitivity to prodrug IAA was examined. As predicted, HRP expressed only in cells transfected with phTERTp-HRP and the expression level after 6 Gy-radiation is much higher than without irradiation. In cells with elevated HRP level, 6 Gy-radiation trigger has shown to render the transfected HEp-2 cells to be more sensitive to IAA-induced killing, which indicated that radio-activation of the promoter did occur and is able to contribute to gene therapy efficacy. The results presented here demonstrate that combining radiotherapy with the hTERT promoter-mediated gene therapy is therefore a promising approach to achieve superior efficacy in targeted cell killing.

The hTERT promoter has been widely used in gene therapy for targeted cancer cells. Combined with radiotherapy, these cancer-targeted gene therapy approaches may achieve a more satisfactory curative effect. The results presented here demonstrate, for the first time, irradiation can be used to enhance the activity of the hTERT promoter. Our data provide support for the hypothesis that the tumor-targeted hTERT promoter can be used to drive transcription of transgenes in the response to DNA-damaging agents, such as irradiation and some chemotherapy drugs.

\section{Acknowledgements}

This study was supported by the National Science Foundation of China (NSFC) Grant No. 30672438 and the Natural Science Foundation of Hubei Province Grant No. 2006ABC009.

\section{References}

1. Kim NW, Piatyszek MA, Prowse KR, et al: Specific association of human telomerase activity with immortal cells and cancer. Science 266: 2011-2015, 1994.

2. Cong YS, Wright WE and Shay JW: Human telomerase and its regulation. Microbiol Mol Biol Rev 66: 407-425, 2002.

3. Gu J and Fang B: Telomerase promoter-driven cancer gene therapy. Cancer Biol Ther 2: S64-S70, 2003.

4. Keith WN, Bilsland A, Hardie M, et al: Drug insight: Cancer cell immortality-telomerase as a target for novel cancer gene therapies. Nat Clin Pract Oncol 1: 88-96, 2004.

5. Hyeon Joo O, Hande MP, Lansdorp PM, et al: Induction of telomerase activity and chromosome aberrations in human tumour cell lines following X-irradiation. Mutat Res 401: 121-131, 1998.
6. Terashima M, Ogawa Y, Toda K, et al: Effects of irradiation on telomerase activity in human lymphoma and myeloma cell lines. Int J Mol Med 2: 567-571, 1998.

7. Neuhof D, Ruess A, Wenz F, et al: Induction of telomerase activity by irradiation in human lymphoblasts. Radiat Res 155 : 693-697, 2001.

8. Schuck A, Poremba C, Lanvers C, et al: Radiation-induced changes of telomerase activity in a human Ewing xenograft tumor. Strahlenther Onkol 178: 701-708, 2002.

9. Zhou F-X, Liao Z-K, Dai J, et al: Radiosensitization effect of zidovudine on human malignant glioma cells. Biochem Biophys Res Commun 354: 351-356, 2007.

10. Horikawa I, Cable PL, Mazur SJ, et al: Downstream E-boxmediated regulation of the human telomerase reverse transcriptase (hTERT) gene transcription: evidence for an endogenous mechanism of transcriptional repression. Mol Biol Cell 13: 2585-2597, 2002.

11. Connolly CN, Futter CE, Gibson A, et al: Transport into and out of the Golgi complex studied by transfecting cells with cDNAs encoding horseradish peroxidase. J Cell Biol 127: 641-652, 1994.

12. Greco O, Folkes LK, Wardman P, et al: Development of a novel enzyme/prodrug combination for gene therapy of cancer: horseradish peroxidase/indole-3-acetic acid. Cancer Gene Ther 7: 1414-1420, 2000.

13. Dachs GU, Tupper J and Tozer GM: From bench to bedside for gene-directed enzyme pro-drug therapy of cancer. Anticancer Drugs 16: 349-359, 2005.

14. Kobayashi S, Sugioka K, Nakano M, et al: Excitation of indole acetate in myeloperoxidase- $\mathrm{H}_{2} \mathrm{O}_{2}$ system: possible formation of indole acetate cation radical. Biochem Biophys Res Commun 93: 967-973, 1980.

15. Folkes LK, Candeias LP and Wardman P: Toward targeted 'oxidation therapy' of cancer: peroxidase-catalysed cytotoxicity of indole-3-acetic acids. Int J Radiat Oncol Biol Phys 42: 917-920, 1998.

16. Stackhouse MA and Buchsbaum DJ: Radiation to control gene expression. Gene Ther 7: 1085-1086, 2000.

17. Datta R, Rubin E, Sukhatme V, et al: Ionizing radiation activates transcription of the EGR1 gene via CArG elements. Proc Natl Acad Sci USA 89: 10149-10153, 1992.

18. Chang JY, Zhang X, Komaki R, et al: Tumor-specific apoptotic gene targeting overcomes radiation resistance in esophageal adenocarcinoma. Int J Radiat Oncol Biol Phys 64: 1482-1494, 2006.

19. Fullerton NE, Boyd M, Ross SC, et al: Comparison of radiohaloanalogues of metaiodobenzylguanidine (MIBG) for a combined gene- and targeted radiotherapy approach to bladder carcinoma. Med Chem 1: 611-618, 2005.

20. Slijepcevic P and Al-Wahiby S: Telomere biology: integrating chromosomal end protection with DNA damage response. Chromosoma 114: 275-285, 2005.

21. Wang X, Liu Y, Chow LS, et al: Regulation of telomerase activity by gamma-radiation in nasopharyngeal carcinoma cells. Anticancer Res 20: 433-437, 2000.

22. Hande MP, Lansdorp PM and Natarajan AT: Induction of telomerase activity by in vivo $\mathrm{X}$-irradiation of mouse splenocytes and its possible role in chromosome healing. Mutat Res 404: 205-214, 1998.

23. Perez Mdel R, Dubner D, Michelin S, et al: Radiation-induced up-regulation of telomerase in KG1a cells is influenced by doserate and radiation quality. Int J Radiat Biol 78: 1175-1183, 2002.

24. Hara T, Noma T, Yamashiro Y, et al: Quantitative analysis of telomerase activity and telomerase reverse transcriptase expression in renal cell carcinoma. Urol Res 29: 1-6, 2001.

25. Sawant SG, Gregoire V, Dhar S, et al: Telomerase activity as a measure for monitoring radiocurability of tumor cells. FASEB J 13: 1047-1054, 1999.

26. Greco O, Patterson AV and Dachs GU: Can gene therapy overcome the problem of hypoxia in radiotherapy? J Radiat Res 41: 201-212, 2000.

27. Greco O, Tozer GM and Dachs GU: Oxic and anoxic enhancement of radiation-mediated toxicity by horseradish peroxidase/indole-3-acetic acid gene therapy. Int J Radiat Biol 78: 173-181, 2002. 